\title{
Kibble-Zurek Mechanism in Driven Dissipative Systems Crossing a Nonequilibrium Phase Transition
}

\author{
A. Zamora, ${ }^{1, *}$ G. Dagvadorj $\odot,{ }^{1,2,{ }^{*}}$ P. Comaron $\odot,{ }^{3,4, *}$ I. Carusotto $\odot,{ }^{5}$ N. P. Proukakis $\odot,{ }^{3}$ and M. H. Szymańska ${ }^{1}$ \\ ${ }^{1}$ Department of Physics and Astronomy, University College London, Gower Street, London WC1E 6BT, United Kingdom \\ ${ }^{2}$ Department of Physics, University of Warwick, Coventry CV4 7AL, United Kingdom \\ ${ }^{3}$ Joint Quantum Centre (JQC) Durham-Newcastle, School of Mathematics, Statistics and Physics, Newcastle University, \\ Newcastle upon Tyne NE1 7RU, United Kingdom \\ ${ }^{4}$ Institute of Physics, Polish Academy of Sciences, Aleja Lotnikw 32/46, 02-668 Warsaw, Poland \\ ${ }^{5}$ INO-CNR BEC Center and Università di Trento, via Sommarive 14, I-38123 Povo, Italy
}

(Received 21 January 2020; accepted 21 July 2020; published 26 August 2020)

\begin{abstract}
The Kibble-Zurek mechanism constitutes one of the most fascinating and universal phenomena in the physics of critical systems. It describes the formation of domains and the spontaneous nucleation of topological defects when a system is driven across a phase transition exhibiting spontaneous symmetry breaking. While a characteristic dependence of the defect density on the speed at which the transition is crossed was observed in a vast range of equilibrium condensed matter systems, its extension to intrinsically driven dissipative systems is a matter of ongoing research. In this Letter, we numerically confirm the Kibble-Zurek mechanism in a paradigmatic family of driven dissipative quantum systems, namely excitonpolaritons in microcavities. Our findings show how the concepts of universality and critical dynamics extend to driven dissipative systems that do not conserve energy or particle number nor satisfy a detailed balance condition.
\end{abstract}

DOI: 10.1103/PhysRevLett.125.095301

One of the most intriguing universal phenomena encountered in the physics of critical systems is the socalled Kibble-Zurek (KZ) mechanism, which successfully describes the spontaneous appearance of long-lived topological defects in complex systems that undergo a spontaneous symmetry breaking when crossing a critical point at a finite speed $[1,2]$. This mechanism is general and spans across vastly different physical realizations as well as length and energy scales, with topological defects ranging from monopoles and vortices to strings and domain walls, depending on the symmetries and the spatial dimensions. In spite of this variety, the density of topological defects has an universal dependence on the rate of change of the control parameter across the transition and on the critical exponents of the system [1-5].

This phenomenon can be physically understood by considering the different stages of critical dynamics when the control parameter is scanned across the critical point. In the initial stages of the dynamics, far from the critical point, the system exhibits an adiabatic behavior, permitted by the fact that the characteristic relaxation time $\tau$ is much shorter than the characteristic time of the control parameter ramp. Later on, since the characteristic relaxation time $\tau$ diverges at the critical point, there must necessarily exist a time after which the system is no longer able to readjust itself adiabatically following the variation of the control parameter and thus enters into a so-called impulse regime. According to the $\mathrm{KZ}$ picture, the density of the topological defects that are left behind at this point of the evolution is determined by the correlation length of the system at this "crossover time" [2].

This KZ mechanism, first proposed in the cosmological context [1,2], has been studied in vastly different contexts spanning across superconducting junction arrays, ion crystals, quantum Ising chains, classical spin systems, holographic superconductors, fermionic and bosonic atomic and helium superfluids, and cosmological scenarios [6-16], with direct experimental confirmations in a broad range of different physical systems [17-31]. A common feature of these studies is that they mostly address cases that are at, or close to, thermal equilibrium and conserve energy and particle number.

Recent experimental progress in the study of excitonpolaritons in semiconductor microcavities embedding quantum wells [32-34] — henceforth referred to as polaritons - has led to hybrid light-matter systems that exhibit a condensation phase transition and the spontaneous appearance of a macroscopic coherence while being inherently in a strongly nonequilibrium condition [32], as the system requires an external pump to compensate for the losses by continuously injecting new polaritons. Polaritons therefore constitute excellent physical platforms to explore the influence that the nonconservation of energy and particle number, and the breaking of the detailed balance condition, may have on the critical dynamics. Pioneering works have started addressing the new features exhibited by the ordered state $[35,36]$, by the 
nonequilibrium phase transition [37-40], the extension of the adiabaticity concept to nonequilibrium scenarios [41-43], the spontaneous formation of defects under a time-dependent pump [44-46], and the late-time relaxation past a sudden quench $[47,48]$.

In this Letter, we investigate the $\mathrm{KZ}$ mechanism in the nonequilibrium phase transition, focusing, in contrast to previous studies [44-48], on the characteristic dependence of the spontaneous vortex nucleation process on the switchon rate of the pump. Our numerical results provide a direct evidence of the adiabatic-to-impulse crossover and confirm the validity of the $\mathrm{KZ}$ picture also in the driven dissipative context of a nonequilibrium phase transition. Compared to a direct study of the number of vortices that are still present at the end of the ramp as a function of the ramp speed, our approach has the key advantage of being insensitive to those vortex annihilation processes that may occur past the critical point [5] and were shown to contribute to the latetime phase ordering dynamics studied in [48].

The key idea for testing and demonstrating the $\mathrm{KZ}$ mechanism is to numerically simulate the dynamical evolution and extract from it the crossover time (subsequently referred to as $-\hat{t}$ ), after which the system is no longer able to adiabatically follow the steady state corresponding to the instantaneous value of the pump. This value is then compared to the corresponding prediction of the $\mathrm{KZ}$ model, i.e., to the time at which the speed of variation of the control parameter starts exceeding the characteristic relaxation time of the system. Similar strategies were previously used for equilibrium scenarios in $[6-8,10]$.

In order to validate the universality of the critical polariton dynamics, we perform two independent calculations for the two most celebrated pumping schemes, which differ in their method of injection and subsequent relaxation processes leading to condensation [32]; specifically, we consider the optical parametric oscillation (OPO) scheme and the incoherent pumping (IP) scheme (see Supplemental Material [49] for details).

Polariton phase transition and modeling.-As discussed in the literature on spontaneous macroscopic coherence and the nonequilibrium condensation phase transition of polaritons $[32,48,50-53,64,65]$, both the OPO and the IP polariton systems show rich yet qualitatively very similar phase diagrams, with two main distinct phases: (i) a disordered phase displaying a low density of polaritons, an exponential decay of spatial correlations and a plasma of unbound vortices and (ii) a (quasi)ordered phase displaying a significant density of polaritons, an algebraic decay of spatial correlations (at least up to relatively long distances $[39,52,54])$, and a low density of vortices, mostly bound in vortex-antivortex pairs $[48,52,65]$.

The intensity of the pump, namely $f_{p}$ (for OPO) and $P$ (for IP), acts as a control parameter and the system is driven from one phase to the other by simply ramping up its value in time at different rates. As usual in condensation phase transitions, the transition from the disordered to the (quasi) ordered phase is accompanied by the breaking of the $\mathrm{U}(1)$ symmetry associated with the phase of the polariton condensate. In the present 2D case, it can be pictorially understood as being mediated by the unbinding of vortexantivortex pairs into a plasma of free vortices $[39,52,66,67]$. A schematic of the phase transition process, depicting our quench sequence and typical initial and final snapshots of the polariton field are shown in Fig. 1.

A powerful way to theoretically describe the collective dynamics of the polariton field across the phase transition is based on a generalized stochastic Gross-Pitaevskii equation. In this model, the nonlinearity arises from the effective polariton-polariton interactions, with suitable additional terms included to describe pumping and losses, and stochastic noise which accounts for the quantum
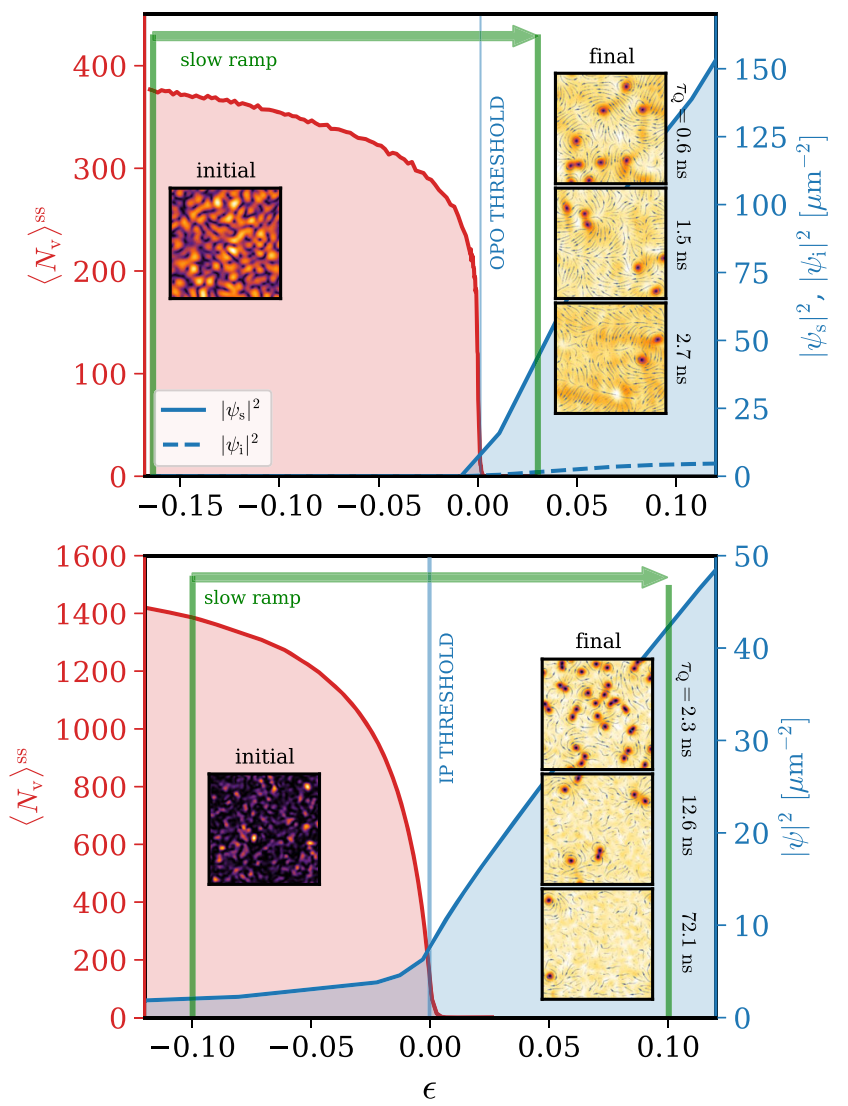

FIG. 1. Nonequilibrium phase transition in the polariton system. Top: OPO case. Steady-state, noise-averaged densities for the signal $\left|\psi_{s}\right|^{2}$ (blue solid line) and idler $\left|\psi_{i}\right|^{2}$ (blue dashed line) fields. Bottom: IP case. Steady-state, noise-averaged field density $|\psi|^{2}$ (blue solid line). In both panels the red curves indicate the steady-state, noise-averaged number of topological defects $\left\langle N_{\mathrm{v}}\right\rangle^{\text {ss }}$. All quantities are plotted as a function of the distance to criticality $\epsilon$. Insets: typical snapshots of the field profile in the initial and final states, as indicated by the thick green arrows at the top of the panels. Typical final state profiles are displayed for different ramp speeds of different timescale $\tau_{Q}$. 
fluctuations [32,36,50,68]. A detailed description of such equations for the polaritons can be found in the Supplemental Material [49]. In order to focus on the intrinsic features of the $\mathrm{KZ}$ physics, we restrict our investigation here to the simplest case of a spatially homogeneous system with periodic boundary conditions.

Ramp protocol.-For the OPO (IP) case, we drive the polariton system through the nonequilibrium phase transition by ramping in time the pump intensity $f_{p}(t)[P(t)]$ across the critical value $f_{p}^{c}\left(P_{c}\right)$, starting from an initial steady state at a pump intensity $f_{p}^{i}\left(P_{i}\right)$ in the disordered phase to a pump intensity $f_{p}^{f}\left(P_{f}\right)$ well in the (quasi)ordered phase. The ramp follows a linear law of characteristic time $\tau_{Q}$. We characterize the phase transition in terms of the distance to criticality, which is quantified by the timedependent parameter $\epsilon(t)$ defined as

$$
\epsilon(t)=\left\{\begin{array}{lc}
\frac{f_{p}^{c}-f_{p}(t)}{f_{p}^{c}}, & (\text { for OPO }) \\
\frac{P(t)-P_{c}}{P_{c}}, & (\text { for IP })
\end{array}\right\}=\varsigma\left(\frac{a_{p}}{\tau_{Q}}\right) t,
$$

where the $a_{p} \equiv\left(f_{p}^{i}-f_{p}^{f}\right) / f_{p}^{c}(\mathrm{OPO})$ or $a_{p} \equiv\left(P_{f}-P_{i}\right) /$ $P_{c}$ (IP) parameter is chosen to have the same value for all ramps within a given pumping scheme and the sign of $\varsigma=\mp 1$ (for OPO or IP) is chosen for consistency with the usual definition of the control parameter in the previous literature on phase transitions. Note that this distinction is required because for the OPO transition we are considering the upper threshold [69], so we need to quench from high to low values of the control parameter. For convenience, we define the origin, $t=0$, of the time axis, as the time when the system crosses the critical point, i.e., $\epsilon(t=0)=0$ based upon $f_{p}(t=0)=f_{p}^{c}(\mathrm{OPO})$ and $P(t=0)=P_{c}$ (IP). Therefore, in both IP and OPO cases, the initial time of the simulation has a negative value, i.e., $t_{i}<0$. Further details of the finite-speed ramp adopted can be found in the Supplemental Material [49].

Testing the KZ mechanism.-First, we need to numerically determine the crossover time $\hat{t}_{\text {num }}$ from the vortex dynamics during a finite-speed ramp. The number of vortices across the Berezinskii-Kosterlitz-Thouless transition at steady state is known to decrease gradually as the transition is approached from the disordered side and to exhibit a sharp decrease in a narrow region around the critical point, as already analyzed for OPO polaritons in $[48,52,64]$. This feature, in combination with a simultaneous study of the spatial correlation function is used to precisely locate the critical point. This behavior is shown for both OPO and IP cases in terms of the distance to criticality $\epsilon$ by the dashed red lines in Fig. 2 (with $\epsilon=0$ denoted by vertical solid lines). When ramping the pump intensity from the disordered phase, the vortex density initially follows the steady-state density during the initial stages of the dynamics, $\epsilon \ll 0$. However, as the dynamical system cannot follow the steady state through the critical point, where the relaxation time diverges, the vortex density eventually departs from its steady-state value, as shown by the solid lines for different ramp timescales $\tau_{Q}$. From this plot we directly extract the numerical crossover time, $\hat{t}_{\text {num }}<0$, at which each of the dynamical curves starts to deviate from the steady-state one. Such times are highlighted for each value of $\tau_{Q}$ by a vertical dashed line in Fig. 2. These lines clearly demonstrate a significant increase in the deviation for smaller values of $\tau_{Q}$, i.e., for faster ramps. More details of the extraction of $\hat{t}_{\text {num }}$ from the data and the dependence of $\hat{t}_{\text {num }}$ on $\tau_{Q}$ can be found in the Supplemental Material [49].

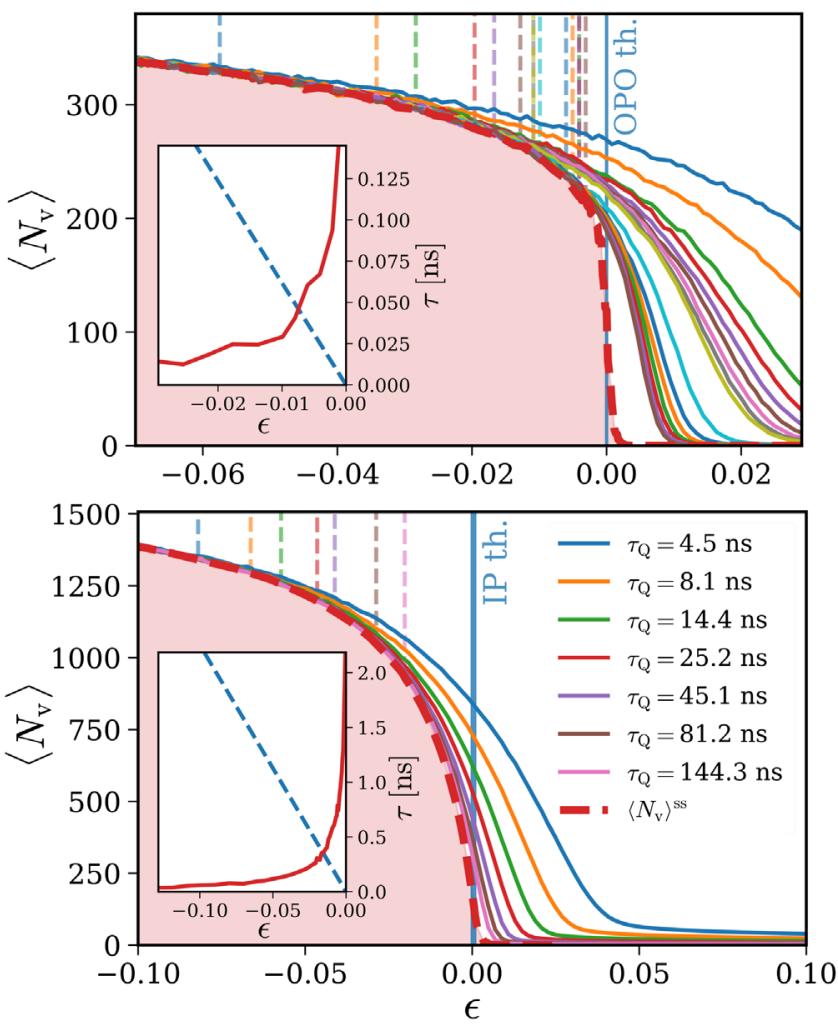

FIG. 2. Average number of vortices as a function of the distance to criticality $\epsilon$ for different ramp speeds of characteristic time $\tau_{Q}$ (thin solid curves) and at steady state (red dashed curves) for OPO (top) and IP (bottom) pumping schemes. Chosen values for OPO (from right to left): $\tau_{Q}=0.3$ (blue curve), 0.6, 0.9, 1.2, 1.5, 1.8, 2.1, 2.4, 2.7, 3, 6, 9, 12, 15, 18, 21, 24 ns (brown curve) (with corresponding IP values indicated within figure). For each value of $\tau_{Q}$, the dashed vertical lines indicate the point $\hat{\epsilon}_{\text {num }}\left(\tau_{Q}\right)$ where the number of vortices in the dynamical evolution starts departing from the steady-state value (see Supplemental Material [49] for details). Insets: red solid curves show the characteristic relaxation time $\tau$ of the vortices as a function of the distance to criticality $\epsilon$. Dashed straight line indicates an example of the dependence of the characteristic time $\epsilon(t) / \dot{\epsilon}(t)$ on $\epsilon$ for a specific choice of ramp parameters, namely $\tau_{Q}=1.2 \mathrm{~ns}$ (OPO) and $\tau_{Q}=4.5 \mathrm{~ns}$ (IP) with $a_{p}=0.1942$ (OPO) and $a_{p}=0.2$ (IP). 
In order to explicitly verify the $\mathrm{KZ}$ mechanism, we should now compare the above numerical prediction for $\hat{t}_{\text {num }}$ with the one extracted by the KZ hypothesis, denoted here by $\hat{t}_{\mathrm{KZ}}$. The KZ hypothesis states that the dynamical results should start departing from the corresponding steady-state ones at the time $\hat{t}_{\mathrm{KZ}}$ at which the relaxation time $\tau$ equals the timescale of the pump variation. Expressed in terms of the distance to criticality $\epsilon(t)$

$$
\tau[\epsilon(t)]_{t=\hat{t}_{\mathrm{KZ}}}=A\left|\frac{\epsilon(t)}{\dot{\epsilon}(t)}\right|_{t=\hat{t}_{\mathrm{KZ}}} .
$$

Here, the dependence of the crossover time $\hat{t}_{\mathrm{KZ}}$ on the ramp speed is contained in the time derivative of the distance to criticality $\epsilon(t)$, and $A$ is a constant parameter of order one. The relaxation time $\tau$ is known to diverge at the critical point, and so the intersection of this with the straight line $\epsilon(t) / \dot{\epsilon}(t)$ defines the crossover time at which the system crosses from an adiabatic to an impulse behavior. This is schematically represented in the insets of Fig. 2. Changing the rate at which the pump intensity is varied will directly affect, via (1), the ramp speed, and thus set a different slope for $\epsilon(t) / \dot{\epsilon}(t)$. Dashed straight lines in the insets of Fig. 2 depict an example of the dependence of the characteristic time $\epsilon(t) / \dot{\epsilon}(t)$ on $\epsilon$ (see caption of Fig. 2 for the exact choice of parameters). Applying this protocol to different values of $\tau_{Q}$ gives the $\mathrm{KZ}$ prediction for $\hat{\epsilon}=\epsilon(\hat{t})$, based on Zurek's expression (2) (with $A=1$ ). In turn, this defines a different intersection point with the relaxation time $\tau(\epsilon)$ in the $\tau(\epsilon)$ vs $\epsilon$ plot (insets of Fig. 2). In order to extract the intersection points for different ramp speeds, we thus need to first extract the system relaxation time $\tau(\epsilon)$ plotted by the solid red line in insets of Fig. 2. For each value of $\epsilon$ in the disordered phase, this is obtained by considering the relaxation time of the number of vortices $N_{v}$ to the steadystate value $N_{v}^{\text {ss }}$ after an infinitely rapid quench of $\epsilon$ toward the desired value,

$$
N_{v}(t)-N_{v}^{\mathrm{ss}} \propto \exp [-t / \tau(\epsilon)]
$$

(see the Supplemental Material [49] for more details).

Validation of $K Z$ mechanism for driven dissipative systems. - The above procedure indicates a linear relation between the numerical $\left(\hat{t}_{\text {num }}\right)$ and the predicted $\left(\hat{t}_{\mathrm{KZ}}\right)$ time for the crossover from adiabatic to impulse behavior, as shown in Fig. 3. We have checked that such a linear relation holds for different choices of the proportionality constant $A$ (beyond $A=1$ ), thus confirming the independence of our conclusions on its specific choice (see Supplemental Material [49] for more details). Since the KZ mechanism is based on the critical properties around the phase transition point, one can naturally expect it to be restricted to sufficiently slow ramps, for which the linear relation is clearly defined. On the other hand, significant deviations from the linear relation between $\hat{t}_{\text {num }}$ and $\hat{t}_{\mathrm{KZ}}$ are expected
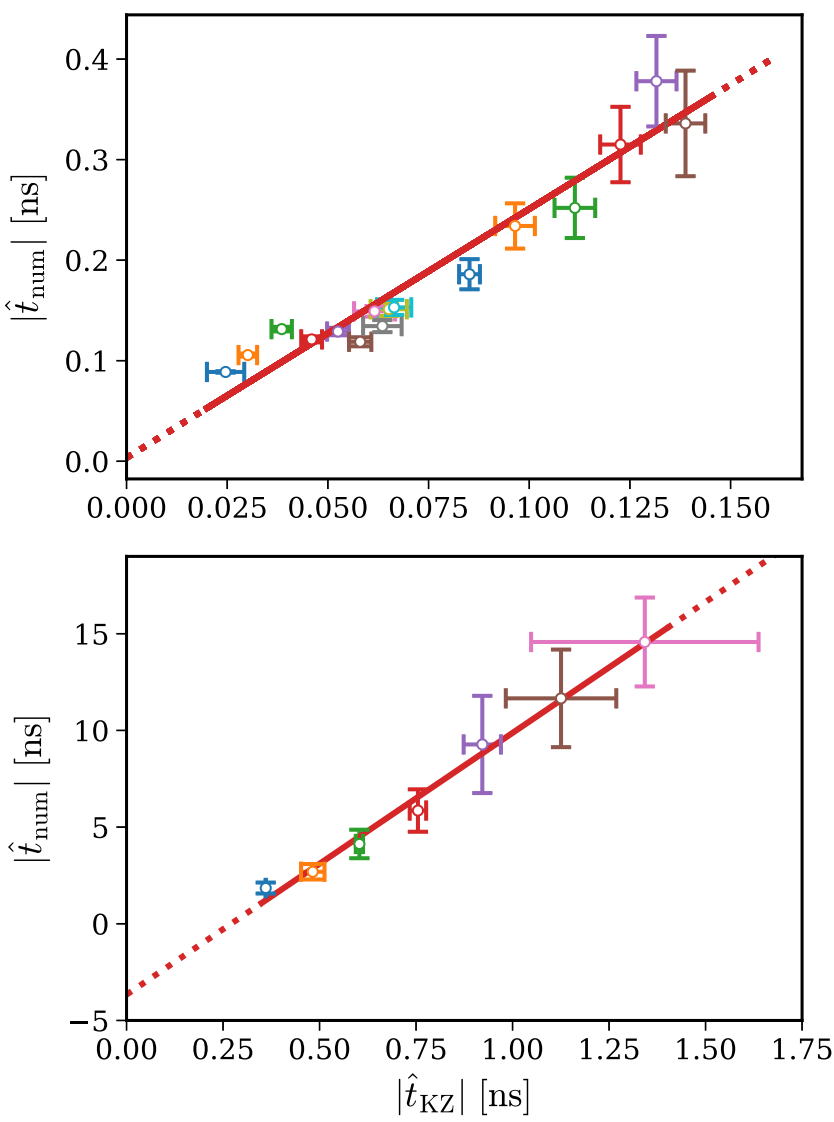

FIG. 3. Numerical prediction for the crossover time $\left|\hat{t}_{\text {num }}\right|$ (corresponding to $\hat{\epsilon}_{\text {num }}$ in Fig. 2) plotted with error bars [70] as a function of the theoretical crossover time $\left|\hat{t}_{\mathrm{KZ}}\right|$ (see insets of Fig. 2) predicted by the Zurek relation Eq. (2) (with $A=1$ ) for the OPO (top) and IP (bottom) pumping schemes. The observed linear dependence between both variables is a clear indication of the applicability of Zurek's relation Eq. (2) and of the KZ mechanism. We obtain a zero intercept (within the error bars) for the OPO polariton system and a small nonzero intercept for the IP case, which indicates nonuniversal subleading order corrections.

to arise for small values of $\hat{t}$, where nonuniversal corrections become important. A hint at such deviations is visible in the presented IP results. Note that the (nonuniversal) intercept of the IP polariton system is highly sensitive to the exact location of the critical point, since a tiny shift in the identification of the critical point within the critical region can shift the intercept toward, or away from, a zero value.

Conclusions. - We have investigated the open question of the extension of the Kibble-Zurek phenomenon to driven dissipative quantum systems. Specifically, we have considered the dynamics of the vortex density during a spontaneous symmetry breaking process across a critical point for a paradigmatic case of a nonequilibrium phase transition, namely the condensation of exciton-polaritons in semiconductor microcavities embedding quantum wells in the strong light-matter coupling regime. Our numerical findings, based on very accurate simulations of the dynamical equations of the systems for experimentally 
relevant parameters, fully confirm the existence of a crossover from an adiabatic to an impulse behavior at a point that depends on the ramp speed and the validity of Zurek's relation [Eq. (2)]. Our analysis thus shows that the $\mathrm{KZ}$ mechanism can maintain its validity even in the case of nonequilibrium phase transitions.

The data that support the findings of this work are available by following the link in [71].

We would like to thank A. Ferrier and M. Matuszewski for fruitful discussions. We acknowledge financial support from the EPSRC (Grants No. EP/I028900/2 and No. EP/ K003623/2) and the Quantera ERA-NET cofund NAQUAS and InterPol projects (EPSRC Grants No. EP/R043434/1 and No. EP/R04399X/1). I. C. acknowledges financial support from the Provincia Autonoma di Trento and from the European Union via the FET-Open Grant "MIR-BOSE" (737017) and the Quantum Flagship Grant "PhoQuS" (820392).

*These authors contributed equally to this work.

[1] T. Kibble, J. Phys. A 9, 1387 (1976).

[2] W. Zurek, Nature (London) 317, 505 (1985).

[3] A. del Campo, T. Kibble, and W. Zurek, J. Phys. Condens. Matter 25, 404210 (2013).

[4] J. Dziarmaga, Adv. Phys. 59, 1063 (2010).

[5] G. Biroli, L. F. Cugliandolo, and A. Sicilia, Phys. Rev. E 81, 050101(R) (2010).

[6] A. Yates and W. H. Zurek, Phys. Rev. Lett. 80, 5477 (1998).

[7] P. Laguna and W. H. Zurek, Phys. Rev. Lett. 78, 2519 (1997).

[8] J. Dziarmaga, J. Meisner, and W. H. Zurek, Phys. Rev. Lett. 101, 115701 (2008).

[9] M. Kolodrubetz, B. K. Clark, and D. A. Huse, Phys. Rev. Lett. 109, 015701 (2012).

[10] A. Jelić and L.F. Cugliandolo, J. Stat. Mech. (2011) $\mathrm{P} 02032$.

[11] J. Sonner, A. del Campo, and W. Zurek, Nat. Commun. 6, 7406 (2015).

[12] P. M. Chesler, A. M. García-García, and H. Liu, Phys. Rev. X 5, 021015 (2015).

[13] P. Silvi, G. Morigi, T. Calarco, and S. Montangero, Phys. Rev. Lett. 116, 225701 (2016).

[14] B. Damski and W. H. Zurek, Phys. Rev. Lett. 104, 160404 (2010).

[15] I.-K. Liu, S. Donadello, G. Lamporesi, G. Ferrari, S.-C. Gou, F. Dalfovo, and N. P. Proukakis, Commun. Phys. 1, 24 (2018).

[16] B. Dóra, M. Heyl, and R. Moessner, Nat. Commun. 10, 2254 (2019).

[17] I. Chuang, R. Durrer, N. Turok, and B. Yurke, Science 251, 1336 (1991).

[18] M. J. Bowick, L. Chandar, E. A. Schiff, and A. M. Srivastava, Science 263, 943 (1994).

[19] P. C. Hendry, N. S. Lawson, R. A. Lee, P. V. E. McClintock, and C. D. H. Williams, Nature (London) 368, 315 (1994).

[20] C. Bäuerle, Y. Bunkov, S. Fisher, H. Godfrin, and G. Pickett, Nature (London) 382, 332 (1996).
[21] V. Ruutu, V. Eltsov, A. Gill, T. Kibble, M. Krusius, Y. G. Makhlin, B. Placais, G. Volovik, and W. Xu, Nature (London) 382, 334 (1996).

[22] A. Maniv, E. Polturak, and G. Koren, Phys. Rev. Lett. 91, 197001 (2003).

[23] K. Pyka et al., Nat. Commun. 4, 2291 (2013).

[24] S. Deutschländer, P. Dillmann, G. Maret, and P. Keim, Proc. Natl. Acad. Sci. U.S.A. 112, 6925 (2015).

[25] L. E. Sadler, J. M. Higbie, S. R. Leslie, M. Vengalattore, and D. M. Stamper-Kurn, Nature (London) 443, 312 (2006).

[26] C. Weiler, T. W. Neely, D. R. Scherer, A. S. Bradley, M. J. Davis, and B.P. Anderson, Nature (London) 455, 948 (2008).

[27] G. Lamporesi, S. Donadello, S. Serafini, F. Dalfovo, and G. Ferrari, Nat. Phys. 9, 656 (2013).

[28] L. Corman, L. Chomaz, T. Bienaime, R. Desbuquois, C. Weitenberg, S. Nascimbene, J. Dalibard, and J. Beugnon, Phys. Rev. Lett. 113, 135302 (2014).

[29] L. Chomaz, L. Corman, T. Bienaimé, R. Desbuquois, C. Weitenberg, S. Nascimbène, J. Beugnon, and J. Dalibard, Nat. Commun. 6, 6162 (2015).

[30] N. Navon, A. L. Gaunt, R. P. Smith, and Z. Hadzibabic, Science 347, 167 (2015).

[31] B. Ko, J. W. Park, and Y. Shin, Nat. Phys. 15, 1227 (2019).

[32] I. Carusotto and C. Ciuti, Rev. Mod. Phys. 85, 299 (2013).

[33] H. Deng, H. Haug, and Y. Yamamoto, Rev. Mod. Phys. 82, 1489 (2010).

[34] J. Kasprzak et al., Nature (London) 443, 409 (2006).

[35] M. H. Szymańska, J. Keeling, and P. B. Littlewood, Phys. Rev. Lett. 96, 230602 (2006).

[36] M. Wouters and I. Carusotto, Phys. Rev. Lett. 99, 140402 (2007).

[37] L. M. Sieberer, M. Buchhold, and S. Diehl, Rep. Prog. Phys. 79, 096001 (2016).

[38] L. M. Sieberer, A. Chiocchetta, A. Gambassi, U. C. Täuber, and S. Diehl, Phys. Rev. B 92, 134307 (2015).

[39] E. Altman, L. M. Sieberer, L. Chen, S. Diehl, and J. Toner, Phys. Rev. X 5, 011017 (2015).

[40] A. Zamora, L. M. Sieberer, K. Dunnett, S. Diehl, and M. H. Szymańska, Phys. Rev. X 7, 041006 (2017).

[41] P. Hedvall and J. Larson, arXiv:1712.01560.

[42] M. Tomka, L. Campos Venuti, and P. Zanardi, Phys. Rev. A 97, 032121 (2018).

[43] W. Verstraelen and M. Wouters, Phys. Rev. A 101, 043826 (2020).

[44] K. Lagoudakis, F. Manni, B. Pietka, M. Wouters, T. C. H. Liew, V. Savona, A. V. Kavokin, R. Andre, and B. DeveaudPledran, Phys. Rev. Lett. 106, 115301 (2011).

[45] M. Matuszewski and E. Witkowska, Phys. Rev. B 89, 155318 (2014).

[46] D. D. Solnyshkov, A. V. Nalitov, and G. Malpuech, Phys. Rev. Lett. 116, 046402 (2016).

[47] M. Kulczykowski and M. Matuszewski, Phys. Rev. B 95, 075306 (2017).

[48] P. Comaron, G. Dagvadorj, A. Zamora, I. Carusotto, N. P. Proukakis, and M. H. Szymańska, Phys. Rev. Lett. 121, 095302 (2018).

[49] See Supplemental Material at http://link.aps.org/ supplemental/10.1103/PhysRevLett.125.095301 for details 
on our pumping schemes, quench protocol, and dynamical equations, along with additional information on the numerical parameter extraction, the validation of the Zurek relation, and a discussion on the scaling relation between crossover time and quench rate, which includes Refs. [10,32-34, 36,37,48,50-63].

[50] I. Carusotto and C. Ciuti, Phys. Rev. B 72, 125335 (2005).

[51] K. Dunnett and M. H. Szymańska, Phys. Rev. B 93, 195306 (2016).

[52] G. Dagvadorj, J. M. Fellows, S. Matyjaśkiewicz, F. M. Marchetti, I. Carusotto, and M. H. Szymańska, Phys. Rev. X 5, 041028 (2015).

[53] A. Chiocchetta and I. Carusotto, Europhys. Lett. 102, 67007 (2013).

[54] J. Keeling et al., in Universal Themes of Bose-Einstein Condensation, edited by N. P. Proukakis, D. W. Snoke, and P. B. Littlewood (Cambridge University Press, Cambridge, England, 2017), p. 205.

[55] P. G. Savvidis, J. J. Baumberg, R. M. Stevenson, M. S. Skolnick, D. M. Whittaker, and J. S. Roberts, Phys. Rev. Lett. 84, 1547 (2000).

[56] A. Altland and B. D. Simons, Condensed Matter Field Theory (Cambridge University Press, Cambridge, England, 2010).

[57] D. Sanvitto et al., Nat. Phys. 6, 527 (2010).

[58] M. Wouters, T. C. H. Liew, and V. Savona, Phys. Rev. B 82, 245315 (2010).

[59] M. Wouters and I. Carusotto, Phys. Rev. Lett. 105, 020602 (2010).
[60] W. H. Nitsche, N. Y. Kim, G. Roumpos, C. Schneider, M. Kamp, S. Hofling, A. Forchel, and Y. Yamamoto, Phys. Rev. B 90, 205430 (2014).

[61] R. Graham, J. Hope, and M. Johnsson, Comput. Phys. Commun. 184, 201 (2013).

[62] P. C. Hohenberg and B. I. Halperin, Rev. Mod. Phys. 49, 435 (1977).

[63] J. Dziarmaga and W. H. Zurek, Sci. Rep. 4, 5950 (2014).

[64] K. Dunnett, A. Ferrier, A. Zamora, G. Dagvadorj, and M. H. Szymańska, Phys. Rev. B 98, 165307 (2018).

[65] D. Caputo et al., Nat. Mater. 17, 145 (2018).

[66] P. Minnhagen, Rev. Mod. Phys. 59, 1001 (1987).

[67] G. Wachtel, L. M. Sieberer, S. Diehl, and E. Altman, Phys. Rev. B 94, 104520 (2016).

[68] M. Wouters and V. Savona, Phys. Rev. B 79, 165302 (2009).

[69] Note that, while in the IP case a stronger pump power generally favors the ordered phase, in the OPO case the latter is restricted to a finite range of pump intensities, and it is therefore often convenient (as done here) to perform the numerical study at the upper threshold [50,52].

[70] Error bars in this figure consider the uncertainty coming from (i) the statistics of the linear regression and (ii) the uncertainty associated with the extraction of the numerical and theoretical crossover times [49].

[71] P. Comaron, A. Zamora, G. Dagvadorj, I. Carusotto, N. P. Proukakis, and M.H. Szyman'ska, Kibble-Zurek mechanism in driven-dissipative systems crossing a nonequilibrium phase transition (2020), https://doi.org/ 10.25405/data.ncl.10029515. 